\title{
Posterior Tibial Slope and a New Morphometric Method With Multiplanar Reconstruction Technique in a Turkish Sample
}

Ismail Eralp Kacmaz ${ }^{1}$, Ali Er ${ }^{2}$, Can Doruk Basa ${ }^{1}$, Vadym Zhamilov ${ }^{1}$, Mustafa Bozdag ${ }^{2}$, Oguzhan Ekizoglu 3

1. Orthopaedics, Tepecik Training and Research Hospital, Izmir, TUR 2. Radiology, Tepecik Training and Research Hospital, Izmir, TUR 3. Forensic Medicine, Tepecik Training and Research Hospital, Izmir, TUR

Corresponding author: Ismail Eralp Kacmaz, kacmazeralp@gmail.com

\section{Abstract}

Aim: The posterior tibial slope (PTS) is important in planning many orthopedic procedures. The aim of the study is to outline a PTS measurement method using multiplanar reconstruction (MPR) in knee computed tomography (CT) images.

Methods: MPR reconstruction was performed on pre-captured CT angio images of 124 patients. A standard tibial axis was created. Then, using reference points, the PTS was measured separately for the medial PTS (MPTS) and lateral PTS (LPTS). To identify an intra- and interobserver error, the technical error of measurement (TEM), relative TEM (rTEM), and coefficient of reliability (R) of the measurement were analyzed.

Results: The study enrolled 124 patients ( 88 males, 36 females) from 18 to 92 years old. The average MPTS $8.63 \pm 2.7^{\circ}$ and LPTS $7.77 \pm 3.1^{\circ}$ were significantly different $(p<0.05)$. However, there was no difference between the sexes ( $p=0.52$ for MPTS; $p=0.9$ for LPTS). The R for intraobserver reliability was 0.942 for the MPTS and 0.943 for the LPTS, and that for interobserver reliability was 0.815 and 0.806 , respectively.

Conclusions: PTS measurement from CT images appears advantageous as it eliminates measurement limitations due to tibial rotation and has high intra- and interobserver consistency.

Categories: Orthopedics

Keywords: arthroplasty, tomography, morphometry, tibia, turkish, slope, technique

\section{Introduction}

The posterior tibial slope (PTS) is the angle between the lateral tibial plateau and longitudinal anatomical axis of the tibia in the sagittal plane [1]. The PTS differs among individuals and these interindividual differences affect the outcomes of orthopedic treatment. The clinical conditions most often affected by a high PTS are anterior cruciate ligament (ACL) injuries and those requiring prostheses. A high PTS increases the likelihood of ACL injury [2-4]. The PTS differs among populations, and these differences are important when designing total knee arthroplasty (TKA) implants [5,6].

PTS affects component positioning in TKA and maintenance of knee biomechanical balance in high tibial osteotomy (HTO) [7-11]. Incorrect component positioning after arthroplasty can lead to early loosening [812]. Instead of using a standard reference value, i.e., a slope of $7^{\circ}$, individual slope measurements that evaluate the medial and lateral plateaus separately cause fewer complications in HTO and unicondylar knee arthroplasty $[8,13,14]$.

The method most commonly used for measuring PTS involves measuring the angle between the lateral tibial plateau and tibial longitudinal anatomical axis using reference points on lateral knee radiographs [14]. Recent improvements in cross-sectional imaging have led to new measurement techniques [15]. Magnetic resonance imaging (MRI) and computed tomography (CT) are now more widely used for morphometric analysis [16]. Imaging data can be stored in hospital databases for use in future investigations. Many researchers have attempted to devise new morphometric techniques based on the analysis of cross-sectional images of different parts of the skeleton [17]. Furthermore, morphometric measurements based on crosssectional images can be standardized because anatomical features are easy to locate and identify on these images, especially when using CT with multiplanar reconstruction (MPR) or three-dimensional (3D) reconstruction [18-22].

This study investigated whether determining the angle between the lateral tibial plateau and longitudinal anatomical axis of the tibia based on CT images with MPR is applicable for assessing the PTS. 


\section{Materials And Methods}

Computed tomography angiography (CTA) images of 165 patients who underwent multidetector lower extremity CTA in our institution from January 2015 to January 2019 were reviewed retrospectively to determine the PTS. Patients were referred for lower extremity CTA because of peripheral vascular pathology. We excluded all patients with a history of surgery or fracture, and those with any kind of implant around the knee $(n=12)$, radiographic signs of osteoarthritis (defined as $\geqslant$ grade III in the Kellgren-Lawrence classification system) $(n=23)$, or poor image quality, mainly due to technical problems or acute trauma around the knee (which can affect image interpretation and measurements; $n=6$ ). Ultimately, the study enrolled 124 patients.

CTA was performed using a 128-slice CT scanner (SOMATOM Definition Edge, Siemens Healthcare, Erlangen, Germany). Scan parameters were $100 \mathrm{kV}, 208 \mathrm{~mA}$, section thickness $1 \mathrm{~mm}$, and reconstruction interval $0.6 \mathrm{~mm}$

All data were transferred from the archive to a workstation (Aquarius workstation, TeraRecon, San Mateo, CA, USA) via internal network connections, providing MPR images. All measurements were performed by one orthopedic surgeon with 10 years of experience and one radiologist with 10 years of experience in musculoskeletal radiology. The measurement parameters used to evaluate PTS are described below.

First, coronal and sagittal MPR images (slice thickness, $1 \mathrm{~mm}$ ) were created from axial source images. Then, the tibia was made parallel to a reference line along the posterior margin of the tibial plateaus in the axial image (Figure 1a) to correct for tibial rotation. The correct axis was formed in the sagittal and coronal images created as a result of MPR. Then, the line passing through the middle section along the longitudinal long axis of the tibia was determined on axial, coronal, and sagittal MPR images (Figures $1 a-1 c$ ). Thus, a standard axis was achieved in all three planes.

Then, by evaluating the sagittal images, the line connecting the highest points of the anterior and posterior tibia was determined in the section where the deepest point of the medial tibial plateau was seen. The medial PTS (MPTS) was determined by measuring the angle between this line and the line perpendicular to the tibial longitudinal axis ( Figure 2a). Similarly, the lateral PTS (LPTS) was measured on sagittal images with minimal lateral tibial plateau convexity (Figure $2 b$ ).

SPSS 22.0 (IBM, Armonk, NY, USA) was used to analyze the variables. A paired-samples t-test was performed to assess differences between the MPTS and LPTS. An independent-samples t-test was used to compare the MPTS and LPTS between groups based on sex. All differences were considered significant when $\mathrm{p}<0.05$. To assess intra- and interobserver error, two observers (orthopedic surgeon and radiologist) re-evaluated the MPTS and LPTS of all patients on CT images during two sessions in two weeks. The intra- (orthopedic surgeon) and interobserver (radiologist) errors were calculated using the technical error of measurement (TEM), relative TEM (rTEM), and coefficient of reliability (R) of the measurement, as suggested by Ulijaszek and Kerr [23].

The protocol for collecting the CTA images of human subjects was approved by the hospital ethics committee, and the study was conducted in accordance with the standards of the Declaration of Helsinki (Finland). 


\section{Cureus}

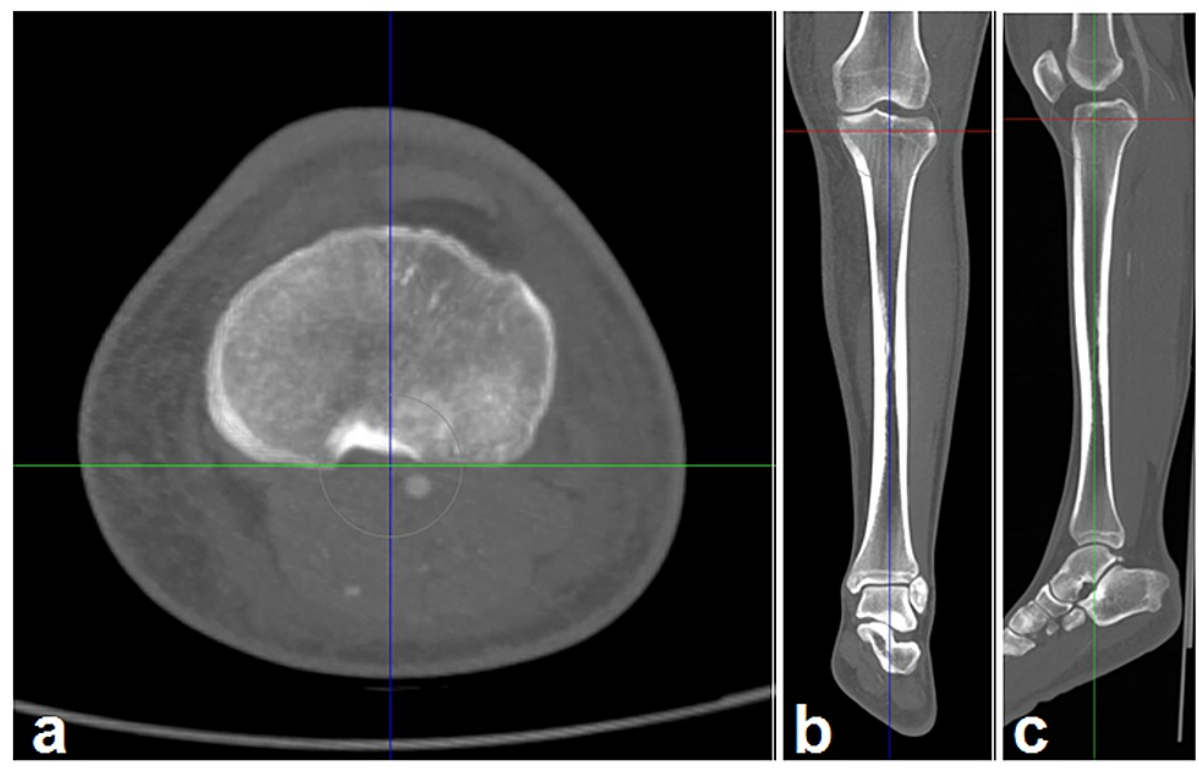

FIGURE 1: Determining the longitudinal axis of the tibia on axial (a), coronal (b), and sagittal (c) images

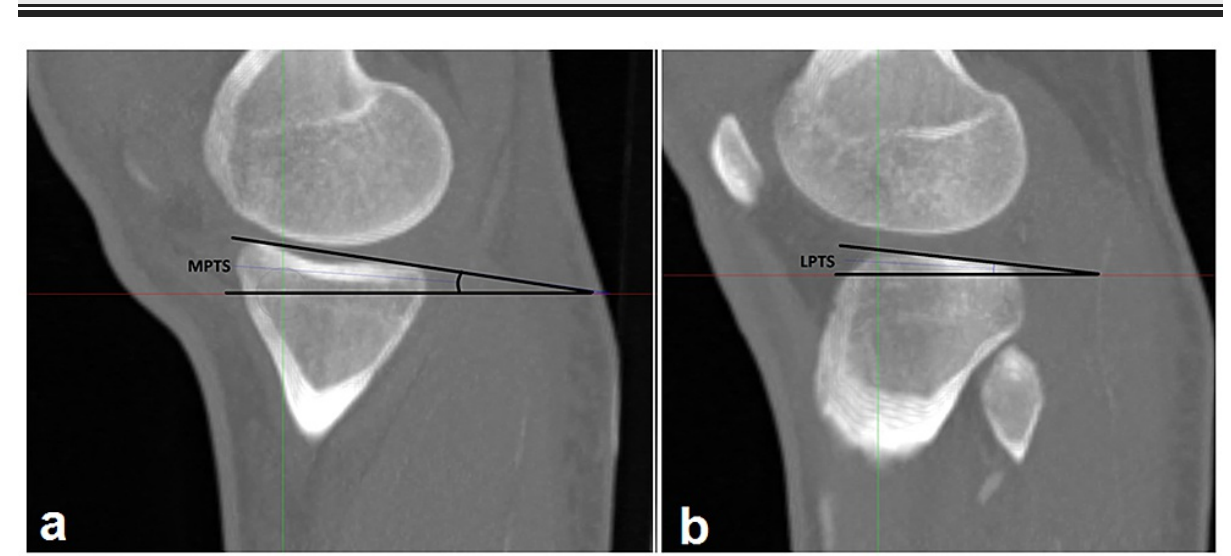

FIGURE 2: Measurement of medial posterior tibial slope (a) and lateral posterior tibial slope (b)

\section{Results}

The study enrolled 124 patients aged 18 to 92 years, including 88 males (mean age $59.9 \pm 13.2$ years) and 36 females (mean age $63.5 \pm 15.1$ years). The average MPTS $8.63 \pm 2.7^{\circ}$ (range 1.3-14.9 ${ }^{\circ}$ ) and LPTS $7.77 \pm 3.1^{\circ}$ (range $\left.1-14.4^{\circ}\right)$ were measured (Table 1 ). The difference between MPTS and LPTS was significant $(\mathrm{p}<0.05)$; however, there was no difference between the sexes in either measurement $(\mathrm{p}=0.52$ for MPTS; $\mathrm{p}=0.9$ for LPTS). 


\section{Cureus}

\begin{tabular}{|c|c|c|c|}
\hline & Mean age (y) & Average MPTS & Average LPTS \\
\hline Female (n:36) & $63.5 \pm 15.1$ & $8.26 \pm 2.9$ & $8.21 \pm 3$ \\
\hline Male (n:88) & $59.9 \pm 13.2$ & $8.78 \pm 2.7^{\circ}$ & $7.59 \pm 3.1^{\circ}$ \\
\hline Total (n:124) & $60.9 \pm 13.8$ & $8.63 \pm 2.7^{\circ}$ & $7.77 \pm 3.1^{\circ}$ \\
\hline
\end{tabular}

\section{TABLE 1: Distribution of average MPTS and LPTS by sex.}

MPTS: medial posterior tibial slope, LPTS: lateral posterior tibial slope, y: years

The intra- and interobserver error rates were also calculated for MPTS and LPTS using TEM, rTEM, and R The intraobserver reliability was $\mathrm{R}=0.942$ for the MPTS and $\mathrm{R}=0.943$ for the LPTS. For the interobserver reliability, the error rates were $\mathrm{R}=0.815$ for the MPTS and $\mathrm{R}=0.806$ for the LPTS. Thus, the intra- and interobserver results were consistent throughout the study (Table 2).

\begin{tabular}{|c|c|c|c|}
\hline & & MPTS & LPTS \\
\hline \multirow{3}{*}{ Intraobserver } & TEM & 0.591 & 0.652 \\
\hline & rTEM & 6.425 & 8.393 \\
\hline & $\mathbf{R}$ & 0.942 & 0.943 \\
\hline \multirow{3}{*}{ Interobserver } & TEM & 1.113 & 1.160 \\
\hline & rTEM & 11.438 & 15.279 \\
\hline & $\mathbf{R}$ & 0.815 & 0.806 \\
\hline
\end{tabular}

\section{TABLE 2: Estimation of intra- and inter-observer error using TEM, rTEM and R.}

TEM, technical error of measurement; rTEM, relative TEM; R; coefficient reliability, MPTS, medial posterior tibial slope; LPTS, lateral posterior tibial slope

\section{Discussion}

Lateral knee radiography is used most commonly for measuring the PTS because of its short duration, low experience requirement, low radiation dose compared to CT, and low cost compared to other methods [1-24]. Many longitudinal axes have been used on lateral knee radiographs for measuring PTS. The lack of a standardized measurement method makes it difficult to compare data and clinical outcomes [6,25]. Measurement error due to tibial rotation can be as high as $13^{\circ}[14,22,26]$. Numerous authors have claimed that both plateaus need to be evaluated separately based on the large differences between cadaver and radiological studies of the PTS $[4,22,27]$. Separate evaluation of the plateaus confers an important advantage in preoperative planning of allograft application after unicondylar knee arthroplasty or tumor resection. Failure to distinguish the lateral and medial plateaus on direct X-rays due to superimposition is a measurement disadvantage $[2,22,24]$. It is thought that the disadvantages of X-ray-dependent measurements can be eliminated by using CT or MRI. Cross-sectional radiological examinations enable 3D analysis and visualization of different sections in one plane as well as better evaluation of cross-sectional morphology with MPR techniques [16,18,20,28,29].

As in measurement methods based on lateral knee radiography, the current CT and MRI studies continue to use different measurement methods. In a 3D-CT study, Zhang et al. measured the MPTS and LPTS using three different methods. They examined points 5 and $15 \mathrm{~cm}$ distal to the tibial plateau in sagittal sections. Three different longitudinal axes passing through the middle of the tibia, running parallel to the anterior cortex and running parallel to the posterior cortex, were identified. The values obtained with each method for all three angles were significantly different from each other [19]. In a CT study, Han et al. identified four different axes [6]. They measured the angles between the mechanical axis (MA), tibial anatomical axis (TAA), anterior tibial cortex (ATC), and fibular shaft axis (FSA) and reported that PTS measurements using CT can be used safely in TKA cases and that measurements made from the lateral tibia plateau may be more useful than those from the medial. The results of both CT studies support the MPTS and LPTS measurement differences and the efficacy of the CT measurement method we present here. 
MRI appears useful for evaluating the medial and lateral tibial plateaus [6]. It requires no ionizing radiation, and the additional soft tissue evaluation can help clinical decisions during measurement. Simon et al. segmented 3D tibia images created using MRI images with AMIRA software (v4.1.2, Visage Imaging, Carlsbad, CA, USA) and measured the PTS using reference points similar to those described by Hashemi et al. $[1,3]$. They used the tibial spine to determine the tibial plateau boundaries. However, the tibial spine differs from person to person. This causes difficulty in determining reference points. With the MRI measurement method used by Hudek et al., cranial and caudal rings drawn according to the anterior and posterior cortex boundaries were identified in the images, and the longitudinal axis was determined based on the rings. Although this was a good technique for determining the longitudinal axis, rotation cannot be excluded because the entire tibia cannot be seen [20]. Our measurement method using CTA eliminates rotation-related limitations as it allows imaging of the entire tibia. The long axis of the tibia is detected exactly by MPR with CTA images. Although this method cannot be used routinely in patients undergoing CTA tibial slope evaluation, it can be used to determine the PTS in patients who require a diagnostic knee CT examination.

Kuwano et al. used the lateral tibial slope as a reference in patients undergoing TKA. They made measurements using 3D-CT, arguing that this is more consistent than measurements made with radiography or MRI. Especially in knees with osteoarthritis, the correct anteroposterior image could not be obtained due to the lack of full extension, so CT measurement was preferable [5].

In their study comparing different imaging methods, Naendrup et al. stated that the main limitation was not being able to exclude the effects of rotation defects, whereas we found that it is possible to minimize the measurement errors caused by rotation by using all axes when applying MPR [30].

Revealing the anatomy in more detail may be important for reducing observer-dependent errors. As in previous CT and MRI studies, our low intra- and interobserver errors may support this. Similarly, the low observer errors support the reliability of this approach in terms of the applicability and reproducibility of the defined methodologies. However, as with any new method, our technique should be evaluated by independent researchers in terms of operator-dependent errors. Operator experience must be considered when standardizing MPR measurements.

Comparing the results of morphometric analyses performed using different imaging techniques can help to refine the methods. Given the unique advantages and disadvantages of the various imaging modalities, studies should aim to help researchers choose the most appropriate method. Since our study was retrospective, different imaging methods could not be applied to the same patient. In addition, except for prospective diagnostic purposes, it is unethical to expose a patient to additional X-ray radiation or CT scans. In 2020, Naendrup et al. evaluated the correlations among radiography, MRI, and CT measurements of PTS [30]. There was a high degree of variability among, and inaccuracy in, the three imaging methods [16]. We recommend computed tomographic detection of parts of the anatomy, given its high sensitivity for determining standard reference points. In clinical practice, it may not be possible to apply our measurement method routinely, but it will be a useful option in some cases, including for arthroplasty, orthopedic oncology (for cases requiring major reconstruction), and revision ACL surgery, as well as in cases in which the evaluation is hampered by poor-quality X-ray images.

The main limitation of the methodology presented here is the ethical issue posed by the high radiation dose, which could be a barrier to its routine use in clinical practice. The image quality and clinical utility of lowdose radiation CT are remarkable. In particular, in anatomical evaluations of bone tissue, the quality of images obtained with low-dose CT may be sufficient for determining relevant anatomical landmarks and axes. Nevertheless, there are no analyses comparing the results with high- and low-dose CT.

In this study, two different observers re-evaluated the data, and the high intra- and interobserver agreement observed supports the reproducibility of the methodology. However, the CT cross-section on which the observers made their measurements was not specified in advance. This limits repeatability since it is not infeasible to use the same cross-section for each measurement. The sections that the observers evaluated, and the effects of difference therein on the results, could not be tested.

In this study, a new method for measuring the PTS based on CT with MPR was introduced. However, as this was a retrospective study, the measurements could not be compared to X-ray or MRI images of the same patients. Different imaging modalities and methods for measuring the PTS yield different values, i.e., there are discrepancies among methods [30].

\section{Conclusions}

Our study obtained valuable data regarding clinical measurement of the PTS. Our method involves CT assessment of the lower extremities and eliminates the negative effects of tibial rotation associated with Xrays. However, it has the disadvantage of increased radiation exposure. Measuring the PTS using CT requires operator experience due to the difficulty in defining landmarks. Nonetheless, retrospective CT with MPR may be clinically useful, minimizing artifacts and allowing the degree of tibial rotation to be determined. 


\section{Additional Information \\ Disclosures}

Human subjects: All authors have confirmed that this study did not involve human participants or tissue. Animal subjects: All authors have confirmed that this study did not involve animal subjects or tissue. Conflicts of interest: In compliance with the ICMJE uniform disclosure form, all authors declare the following: Payment/services info: All authors have declared that no financial support was received from any organization for the submitted work. Financial relationships: All authors have declared that they have no financial relationships at present or within the previous three years with any organizations that might have an interest in the submitted work. Other relationships: All authors have declared that there are no other relationships or activities that could appear to have influenced the submitted work.

\section{Acknowledgements}

The English in this document has been checked by at least two professional editors, both native speakers of English. For a certificate, please see: http://www.textcheck.com/certificate/XTbpan.

\section{References}

1. Hashemi J, Chandrashekar N, Gill B, et al.: The geometry of the tibial plateau and its influence on the biomechanics of the tibiofemoral joint. J Bone Joint Surg Am. 2008, 90:2724-34. 10.2106/JBJS.G.01358

2. Jenny JY, Boeri C, Ballonzoli L, Meyer N: Difficulties and reproducibility of radiological measurement of the proximal tibial axis according to Levigne. (Article in French). Rev Chir Orthop Reparatrice Appar Mot. 2005, 91:658-63. 10.1016/s0035-1040(05)84470-8

3. Simon RA, Everhart JS, Nagaraja HN, Chaudhari AM: A case-control study of anterior cruciate ligament volume, tibial plateau slopes and intercondylar notch dimensions in ACL-injured knees. J Biomech. 2010, 43:1702-7. 10.1016/j.jbiomech.2010.02.033

4. Stijak L, Herzog RF, Schai P: Is there an influence of the tibial slope of the lateral condyle on the ACL lesion? A case-control study. Knee Surg Sports Traumatol Arthrosc. 2008, 16:112-7. 10.1007/s00167-0070438-1

5. Kuwano T, Urabe K, Miura H, et al.: Importance of the lateral anatomic tibial slope as a guide to the tibial cut in total knee arthroplasty in Japanese patients. J Orthop Sci. 2005, 10:42-7. 10.1007/s00776-004-0855-7

6. Han H, Oh S, Chang CB, Kang SB: Anthropometric difference of the knee on MRI according to gender and age groups. Surg Radiol Anat. 2016, 38:203-11. 10.1007/s00276-015-1536-2

7. Stulberg SD: How accurate is current TKR instrumentation?. Clin Orthop Relat Res. 2003, 177-84. 10.1097/01.blo.0000093029.56370.0f

8. Hofmann AA, Bachus KN, Wyatt RW: Effect of the tibial cut on subsidence following total knee arthroplasty. Clin Orthop Relat Res. 1991, 63-9.

9. Singerman R, Dean JC, Pagan HD, Goldberg VM: Decreased posterior tibial slope increases strain in the posterior cruciate ligament following total knee arthroplasty. J Arthroplasty. 1996, 11:99-103.

10. Waelchli B, Romero J: Dislocation of the polyethylene inlay due to anterior tibial slope in revision total knee arthroplasty. Knee Surg Sports Traumatol Arthrosc. 2001, 9:296-8. 10.1007/s001670100203

11. Klek M, Dhawan A: The role of high tibial osteotomy in ACL reconstruction in knees with coronal and sagittal plane deformity. Curr Rev Musculoskelet Med. 2019, 12:466-71. 10.1007/s12178-019-09589-9

12. Catani F, Leardini A, Ensini A, Cucca G, Bragonzoni L, Toksvig-Larsen S, Giannini S: The stability of the cemented tibial component of total knee arthroplasty: posterior cruciate-retaining versus posteriorstabilized design. J Arthroplasty. 2004, 19:775-82. 10.1016/j.arth.2004.01.013

13. Hernigou P, Deschamps G: Posterior slope of the tibial implant and the outcome of unicompartmental knee arthroplasty. J Bone Joint Surg Am. 2004, 86:506-11. 10.2106/00004623-200403000-00007

14. Faschingbauer M, Sgroi M, Juchems M, Reichel H, Kappe T: Can the tibial slope be measured on lateral knee radiographs?. Knee Surg Sports Traumatol Arthrosc. 2014, 22:3163-7. 10.1007/s00167-014-2864-1

15. Smith-Bindman R, Miglioretti DL, Larson EB: Rising use of diagnostic medical imaging in a large integrated health system. Health Aff (Millwood). 2008, 27:1491-502. 10.1377/hlthaff.27.6.1491

16. Hanazawa T, Sano T, Seki K, Okano T: Radiologic measurements of the mandible: a comparison between CT-reformatted and conventional tomographic images. Clin Oral Implants Res. 2004, 15:226-32. 10.1111/j.1600-0501.2004.00991.x

17. Kaneko Y, Matsumoto M, Takaishi H, Nishiwaki Y, Momoshima S, Toyama Y: Morphometric analysis of the lumbar intervertebral foramen in patients with degenerative lumbar scoliosis by multidetector-row computed tomography. Eur Spine J. 2012, 21:2594-602. 10.1007/s00586-012-2408-7

18. Zhang Y, Chen Y, Qiang M, Zhang K, Li H, Jiang Y, Jia X: Comparison between three-dimensional CT and conventional radiography in proximal tibia morphology. Medicine (Baltimore). 2018, 97:e11632. 10.1097/MD.0000000000011632

19. Zhang Y, Wang J, Xiao J, Zhao L, Li ZH, Yan G, Shi ZJ: Measurement and comparison of tibial posterior slope angle in different methods based on three-dimensional reconstruction. Knee. 2014, 21:694-8. 10.1016/j.knee.2014.01.008

20. Hudek R, Schmutz S, Regenfelder F, Fuchs B, Koch PP: Novel measurement technique of the tibial slope on conventional MRI. Clin Orthop Relat Res. 2009, 467:2066-72. 10.1007/s11999-009-0711-3

21. Lipps DB, Wilson AM, Ashton-Miller JA, Wojtys EM: Evaluation of different methods for measuring lateral tibial slope using magnetic resonance imaging. Am J Sports Med. 2012, 40:2731-6. 10.1177/0363546512461749

22. Kessler MA, Burkart A, Martinek V, Beer A, Imhoff AB: Development of a 3-dimensional method to determine the tibial slope with multislice-CT. (Article in German). Z Orthop Ihre Grenzgeb. 2003, 141:143-7. $10.1055 / \mathrm{s}-2003-38658$ 


\section{Cureus}

23. Ulijaszek SJ, Kerr DA: Anthropometric measurement error and the assessment of nutritional status . Br J Nutr. 1999, 82:165-77. 10.1017/s0007114599001348

24. Dejour $\mathrm{H}$, Bonnin M: Tibial translation after anterior cruciate ligament rupture. Two radiological tests compared. J Bone Joint Surg Br. 1994, 76:745-9. 10.1302/0301-620x.76b5.8083263

25. Noyes FR, Goebel SX, West J: Opening wedge tibial osteotomy: the 3-triangle method to correct axial alignment and tibial slope. Am J Sports Med. 2005, 33:378-87. 10.1177/0363546504269034

26. Lonner JH, Laird MT, Stuchin SA: Effect of rotation and knee flexion on radiographic alignment in total knee arthroplasties. Clin Orthop Relat Res. 1996, 102-6. 10.1097/00003086-199610000-00014

27. Yoo JH, Chang CB, Shin KS, Seong SC, Kim TK: Anatomical references to assess the posterior tibial slope in total knee arthroplasty: a comparison of 5 anatomical axes. J Arthroplasty. 2008, 23:586-92. 10.1016/j.arth.2007.05.006

28. Yue B, Varadarajan KM, Ai S, Tang T, Rubash HE, Li G: Differences of knee anthropometry between Chinese and white men and women. J Arthroplasty. 2011, 26:124-30. 10.1016/j.arth.2009.11.020

29. Utzschneider S, Goettinger M, Weber P, Horng A, Glaser C, Jansson V, Müller PE: Development and validation of a new method for the radiologic measurement of the tibial slope. Knee Surg Sports Traumatol Arthrosc. 2011, 19:1643-8. 10.1007/s00167-011-1414-3

30. Naendrup JH, Drouven SF, Shaikh HS, Jaecker V, Offerhaus C, Shafizadeh ST, Pfeiffer TR: High variability of tibial slope measurement methods in daily clinical practice: comparisons between measurements on lateral radiograph, magnetic resonance imaging, and computed tomography. Knee. 2020, 27:923-9.

10.1016/j.knee.2020.01.013 